\title{
Interstellar Broadening of Images in the Gravitational Lens PKS 1830-211
}

\author{
D.L. Jones, R.A. Preston, D.W. Murphy \\ Jet Propulsion Laboratory, California Institute of Technology, Pasadena, CA. 91109 \\ D.L. Jauncey, J.E. Reynolds, A.K. Tzioumis, E.A. King \\ Australia Telescope National Facility, CSIRO, Epping, NSW, Australia \\ P.M. McCulloch, J.E.J. Lovell, M.E. Costa \\ Dept. of Physics, University of Tasmania, Hobart, Tasmania, Australia \\ and \\ T.D. van Ommen \\ Antarctic CRC, University of Tasmania, Hobart, Tasmania, Australia
}

Received __; accepted _ 


\begin{abstract}
The remarkably strong radio gravitational lens PKS 1830-211 consists of a one arcsecond diameter Einstein ring with two bright compact (milliarcsecond) components located on opposite sides of the ring. We have obtained $22 \mathrm{GHz}$ VLBA data on this source to determine the intrinsic angular sizes of the compact components. Previous VLBI observations at lower frequencies indicate that the brightness temperatures of these components are significantly lower than $10^{10} \mathrm{~K}$ (Jauncey, et al. 1991), less than is typical for compact synchrotron radio sources and less than is implied by the short timescales of flux density variations. A possible explanation is that interstellar scattering is broadening the apparent angular size of the source and thereby reducing the observed brightness temperature. Our VLBA data support this hypothesis. At $22 \mathrm{GHz}$ the measured brightness temperature is at least $10^{11} \mathrm{~K}$, and the deconvolved size of the core in the southwest compact component is proportional to $\nu^{-2}$ between 1.7 and $22 \mathrm{GHz}$. VLBI observations at still higher frequencies should be unaffected by interstellar scattering.
\end{abstract}

Subject headings: galaxies: individual (PKS 1830-211) — gravitational lensing - scattering 


\section{Introduction}

The Einstein ring gravitational lens PKS 1830-211 is one of the strongest compact radio sources in the sky, nearly two orders of magnitude brighter at radio frequencies than any other known gravitational lens (Rao and Subrahmanyan 1988; Jauncey, et al. 1991). The source consists of a pair of bright compact (mas) components on opposite sides of a 1 arcsecond diameter ring of emission. Both compact components have been imaged with VLBI at $1.7 \mathrm{GHz}$ (Jones, unpublished), $2.3 \mathrm{GHz}$ (Jauncey, et al. 1991; King, et al. 1993), 5 GHz (Jones, et al. 1993; Jones 1994), and 22 GHz (Jones, et al. 1995 and this paper). Variations in the morphology of both compact components have been seen between two VLBI epochs at $5 \mathrm{GHz}$ (Jones 1994). The extragalactic nature of PKS 1830-211 was confirmed by galactic HI emission and absorption measurements (Subrahmanyan, Kesteven, and te Lintel Hekkert 1992).

The large radio flux density of PKS 1830-211 has allowed a large quantity of high signal-to-noise single-dish and interferometric data to be obtained (e.g., Subrahmanyan, et al. 1990; Jauncey, et al. 1992, 1993; van Ommen, et al. 1995; Lovell, et al. 1995). This wealth of observational information provides strong constraints on the geometry of the lens system and may lead to an accurate estimate of the large-scale value of $\mathrm{H}_{\circ}$ when combined with redshifts and the differential time delay between the two images.

The over-all morphology of the source has been successfully modeled as a background core-jet radio source lensed by a single medium-mass galaxy (Kochanek and Narayan 1992; Nair, Narasimha, and Rao 1993). An optical redshift for the background source is still unavailable because the position of PKS $1830-211$ is close to the galactic center $(l=12.2$, $b=-5.7$ ) in a region crowded with foreground stars (Djorgovsky, et al. 1992; Jauncey,

et al. 1993). However, the redshift of the lensing galaxy has been recently determined from radio absorption line observations (Wiklind and Combes 1996). The time delay 
between the two compact components has been estimated as $44 \pm 9$ days by van Ommen, et al. (1995) based on multi-epoch VLA images. Proposed observations with the VSOP spacecraft could improve the accuracy of the time delay determination.

One of the aspects of this source which has been difficult to explain is the unusually low brightness temperatures of the two compact components. The total flux density varies by factors of more than 2 on time scales of months and years (Lovell, et al. 1995), which implies a smaller angular size and higher brightness temperature than seen in VLBI images at frequencies $\leq 5 \mathrm{GHz}$. Since the line of sight to PKS 1830-211 passes close to the galactic center, it is plausible that significant interstellar scattering (ISS) within our galaxy may occur along this line of sight. Scattering by the interstellar medium in the lensing galaxy will be far less significant than ISS within our galaxy (Walker 1996). The effects of ISS decrease rapidly with frequency, and should be significantly smaller at $22 \mathrm{GHz}$.

\section{Observations}

We observed PKS 1830-221 with the full VLBA at $22 \mathrm{GHz}$ in May 1994. The data were correlated in Socorro twice using phase centers corresponding to the locations of the two compact components, which we designate the northeast (NE) and southwest (SW) components. The observations were inadvertently made in dual-polarization mode, which resulted in much less suppression of the component far from the phase center (by decorrelation over the observing bandwidth) than expected. As a result, it was necessary to include both compact components in the imaging process. After manual phase calibration, amplitude calibration, and fringe fitting in AIPS円, we used the Caltech program

\footnotetext{
${ }^{1}$ The Astronomical Image Processing System was developed by the National Radio Astronomy Observatory, which is operated by Associated Universities, Inc., under a
} 
Difmap (Shepherd, Pearson, and Taylor 1994) for editing, self-calibration, imaging, and deconvolution. The data were coherently averaged to 15 seconds, and the errors were estimated from the scatter of the 1-second data points within each averaging interval.

Phase-only corrections were applied during each cycle of self-calibration, Fourier inversion, and deconvolution until the model flux density matched that of the visibility data on the shortest baselines to within a few percent. At that point time-independent antenna gain corrections were allowed (these corrections were $\leq 12 \%$ for all antennas) for several cycles, followed by time-dependent gain corrections with decreasing time scales until full point-by-point amplitude self-calibration was applied. The self-calibration solutions remained very stable from one cycle to the next. The final agreement factor (reduced $\chi^{2}$ ) between our model and the self-calibrated data was 0.86 .

\section{Results}

Figures 1 and 2 show our VLBA images of the NE and SW components of PKS 1830211, respectively. The NE component appears to have a jet extending at least 12 mas towards the NW, and a bright core that is slightly extended towards the NW as well. The SW component appears to be very nearly unresolved. Recent $15 \mathrm{GHz}$ VLBI observations by Garrett, et al. (1995) confirm that the NE component has a well-defined jet as well as a core, while the SW component is essentially unresolved (see also Patnaik and Porcas 1995). The SW component in PKS 1830-211 is the more compact of the two bright components at all frequencies, and consequently is the best one to use as a test for ISS broadening. The 22-GHz image of the SW component shown in Jones, et al. (1995) has slightly higher angular resolution but significantly lower dynamic range than the image in figure 2 .

cooperative agreement with the National Science Foundation. 
EDITOR: PLACE FIGURE 1 HERE.

\section{EDITOR: PLACE FIGURE 2 HERE.}

The angular separation between the brightest peaks in figures 1 and 2 is $972 \pm 1$ mas. VLBI observations of PKS 1830-211 at 5 GHz in November 1990 and September 1991 indicated angular separations of $975 \pm 2$ and $973 \pm 2$ mas, respectively (Jones, et al. 1993). Thus, we find no evidence for a significant change in separation over 3.5 years. As Williams and Saha (1995) point out, centroid shifts caused by microlensing should not be detectable in radio images.

Figure 3 show the deconvolved minor axis width of the SW component at four frequencies: $1.7 \mathrm{GHz}$ (unpublished data from an ad-hoc VLBA experiment in 1990), 2.3 GHz (King 1995), $4.9 \mathrm{GHz}$ (Jones 1994), and $22 \mathrm{GHz}$ (this paper). Deconvolved sizes are used to remove (or at least reduce) the bias in observed angular sizes which is introduced by the limited range of baseline lengths in each VLBI array. We compare the minor axis sizes because intrinsic source structure is more likely to contribute to the major axis size. The slope of the line fit to the angular size measurements in figure 3 is $1.96 \pm 0.14$, consistent with the $\lambda^{2}$ dependence expected for scattering. This suggests that angular size measurements made by VLBI at frequencies $\leq 22 \mathrm{GHz}$ are indeed affected by angular broadening due to ISS.

\section{EDITOR: PLACE FIGURE 3 HERE.}

At $22 \mathrm{GHz}$ the deconvolved size of the SW component core is less than $0.6 \times 0.2$ mas and $\mathrm{T}_{\mathrm{b}} \geq 10^{11} \mathrm{~K}$. This is much more typical of the brightness temperatures seen in other extragalactic compact radio sources. 


\section{Discussion}

The amount of ISS expected for a line of sight passing close to the center of our galaxy can be estimated from the measured angular broadening of isolated $\mathrm{H}_{2} \mathrm{O}$ maser features in W49(N) and Sgr B2. Gwinn, Moran, and Reid (1988) found minimum angular sizes of 0.2 mas in W49 and 0.3 mas in Sgr B2. This is consistent with our measured angular size of the SW component core in PKS 1830-211 at 22 GHz. Gwinn, Moran, and Reid also found that OH masers in W49 and Sgr B2 had angular sizes consistent with $\Theta \propto \lambda^{2}$ when compared with $\mathrm{H}_{2} \mathrm{O}$ maser sizes in those objects.

It is interesting to compare our measurements with those predicted by the Blandford and Narayan (1985) formula for ISS:

$$
\Theta_{\text {ISS }} \sim 2\left(C_{n}^{2}\right)^{3 / 5} \lambda^{11 / 5} D^{3 / 5} \text { arcseconds, }
$$

where $\lambda$ is the wavelength in meters and $D$ is the path length through the ISM in kpc. $C_{n}^{2}$ gives the strength of electron density fluctuations in the ISM. Anantharamaiah and Narayan (1988) find $C_{n}^{2} \approx 1.5$ for low galactic latitudes and $|l| \leq 30^{\circ}$; we use a value of 1 since the galactic latitude of PKS 1830-211 (-5.7) is not very small. Setting $\lambda=0.013$ meters and $D \approx 5 \mathrm{kpc}$ (the path length for $|b|=5.7$ through a plane parallel ISM with a height of $500 \mathrm{pc}$ ) gives us $\Theta_{\text {ISS }} \approx 0.4$ mas. This is also consistent with our angular size determination for PKS 1830-211.

Future work may allow this analysis to be extended to the core of the NE component. This will be more difficult because of the higher level of extended emission associated with the NE component, particularly very close to the brightest peak. If we can determine the degree of angular broadening of the NE component core due to ISS we will have an opportunity to study differences in the scattering properties of our galaxy's ISM at multiple frequencies along two lines of sight one arcsecond apart. The results presented here also 
suggest that still higher frequency VLBI observations of PKS 1830-211 will be useful, since the intrinsic angular size of the radio source core is still not causing a significant deviation from the $\theta \propto \lambda^{2}$ relation at $22 \mathrm{GHz}$.

This research was carried out at the Jet Propulsion Laboratory, California Institute of Technology, under contract with the National Aeronautics and Space Administration. The Australia Telescope is operated as a national facility by CSIRO. 


\section{REFERENCES}

Anantharamaiah, K.R. and Narayan, R. 1988 in Radio Wave Scattering in the Interstellar Medium, ed. J. Cordes, B. Rickett, and D. Backer (AIP: New York), 185

Blandford, R. and Narayan, R. 1985, MNRAS, 213, 591

Djorgovski, S., Meylan, G., Klemola, A., Thompson, D.J., Weir, W.N., Swarup, G., Rao, A.P., Subrahmanyan, R., and Smette, A. 1992, MNRAS, 257, 240

Garrett, M.A., Nair, S., Porcas, R.W., and Patnaik, A.R. 1995, in Astrophysical Applications of Grav. Lensing, ed. C. Kochanek and J. Hewitt (Kluwer), 189

Gwinn, C.R., Moran, J.M., and Reid, M.J. 1988, in Radio Wave Scattering in the Interstellar Medium, ed. J. Cordes, B. Rickett, and D. Backer (AIP: New York), 129

Jauncey, D.L., et al. 1991, Nature, 352, 132

Jauncey, D.L., et al. 1992, in Gravitational Lenses, ed. R. Kayser, T. Schramm, and L. Nieser (Springer Verlag: Berlin), 333

Jauncey, D.L., et al. 1993, in Sub-Arcsecond Radio Astronomy, ed. R. Davis and R. Booth (Cambridge: Cambridge Univ. Press), 134

Jones, D.L., et al. 1993, in Sub-Arcsecond Radio Astronomy, ed. R. Davis and R. Booth (Cambridge: Cambridge Univ. Press), 150

Jones, D.L. 1994, in Compact Radio Sources, ed. J.A. Zensus and K.I. Kellermann (Socorro: NRAO), 135

Jones, D.L., Preston, R.A., Murphy, D.W., Meier, D.L., Jauncey, D.L., Reynolds, J.E., and Tzioumis, A.K. 1995, in Astrophysical Applications of Gravitational Lensing, ed. C. Kochanek and J. Hewitt (Kluwer), 345

King, E.A., et al. 1993, in Sub-Arcsecond Radio Astronomy, ed. R. Davis and R. Booth (Cambridge: Cambridge Univ. Press), 152 
King, E.A. 1995, Ph.D. Thesis, Univ. of Tasmania

Kochanek, C.S. and Narayan, R. 1992, ApJ, 401, 461

Lovell, J.E.J., McCulloch, P.M., King, E.A., and Jauncey, D.L. 1995, in Astrophysical Applications of Grav. Lensing, ed. C. Kochanek and J. Hewitt (Kluwer), 347

Nair, S., Narasimha, D., and Rao, P. 1993, ApJ, 407, 46

Patnaik, A.R. and Porcas, R.W. 1995, in Astrophysical Applications of Gravitational Lensing, ed. C. Kochanek and J. Hewitt (Kluwer), 305

Rao, A.P. and Subrahmanyan, R. 1988, MNRAS, 231, 229

Shepherd, M.C., Pearson, T.J., and Taylor, G.B. 1994, BAAS, 26, 987

Subrahmanyan, R., Narasimha, D., Rao, A.P., and Swarup, G. 1990, MNRAS, 246, 263

Subrahmanyan, R., Kesteven, M.J., and te Lintel Hekkert, P. 1992, MNRAS, 259, 63

van Ommen, T.D., Jones, D.L., Preston, R.A., and Jauncey, D.L. 1995, ApJ, 444, 561

Walker, M. 1996, in Extragalactic Radio Sources (Kluwer), in press.

Wiklind, T. and Combes, F. 1996, Nature, 379, 139

Williams, L.L.R. and Saha, P. 1995, AJ, 110, 1471 
Fig. 1.- VLBA image of the NE compact component in PKS 1830-211 at $22 \mathrm{GHz}$. The contours are $-1,1,2,4,8,16,32,50,70$, and $95 \%$, and the restoring beam (shown in the bottom left corner) is an elliptical Gaussian with FWHM of $2.2 \times 1.1$ mas and the major axis along position angle $1.5^{\circ}$. The absolute flux density scale is not well calibrated, but this changes only the peak brightness level of the image and not the morphology of the source.

Fig. 2.- VLBA image of the SW compact component in PKS 1830-211 at 22 GHz. The contours are $-0.5,0.5,1,2,4,8,16,32,50,70$, and $95 \%$, and the restoring beam is the same as in figure 1.

Fig. 3.- The deconvolved minor axis angular size of the SW component core at $22 \mathrm{GHz}$ $(\lambda=1.3 \mathrm{~cm}), 5 \mathrm{GHz}(\lambda=6 \mathrm{~cm}), 2.3 \mathrm{GHz}(\lambda=13 \mathrm{~cm})$, and $1.7 \mathrm{GHz}(\lambda=18 \mathrm{~cm})$. The deconvolved sizes and their (formal) errors at 22, 5, and $1.7 \mathrm{GHz}$ were determined with the AIPS program IMFIT, using only the upper $50 \%$ of the brightness range to avoid bias by any extended low-level structure. The size at $2.3 \mathrm{GHz}$ is taken from King (1995). A least-squares linear fit to the data points is shown; this line has a slope of $1.96 \pm 0.14$. 


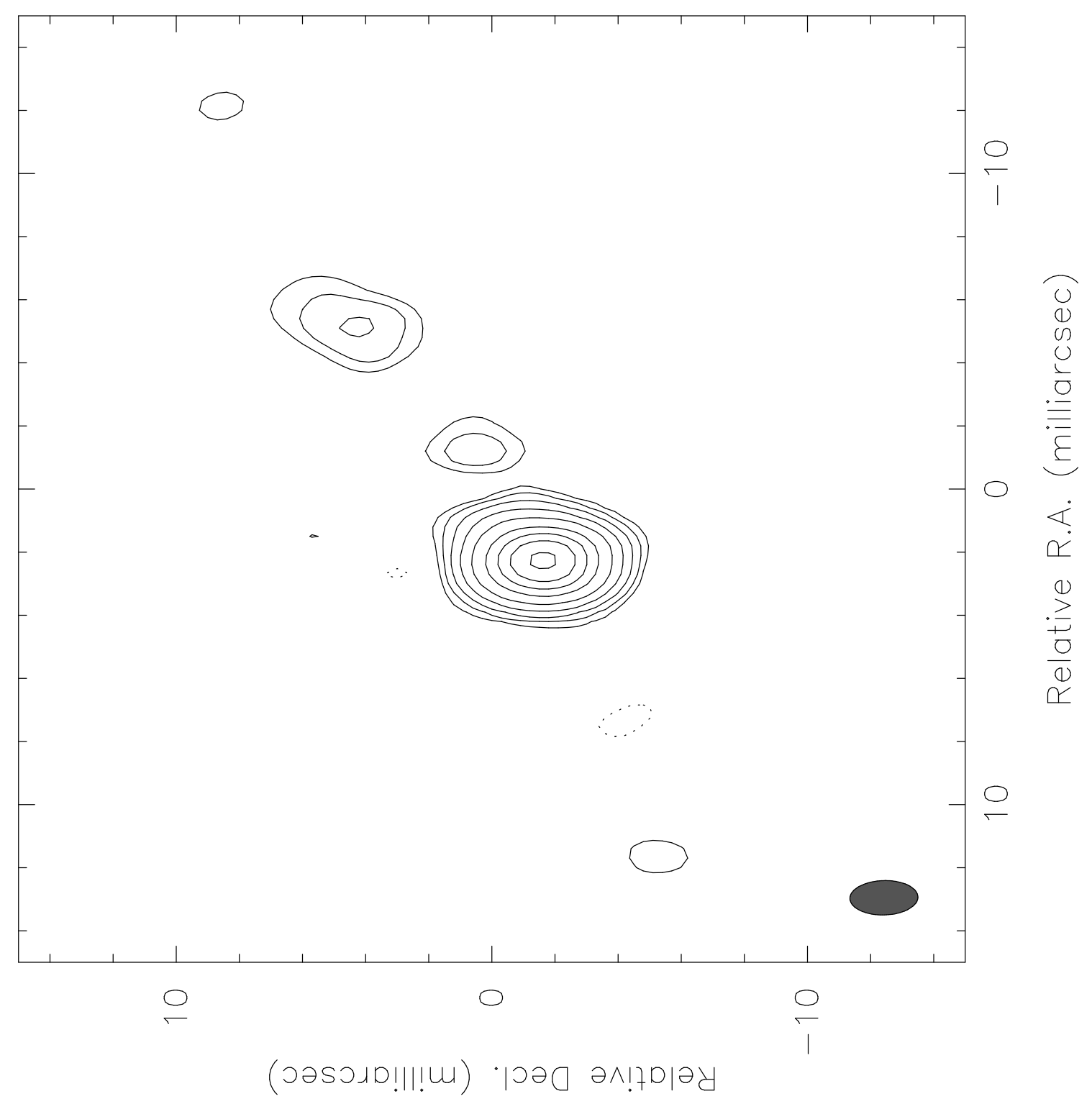




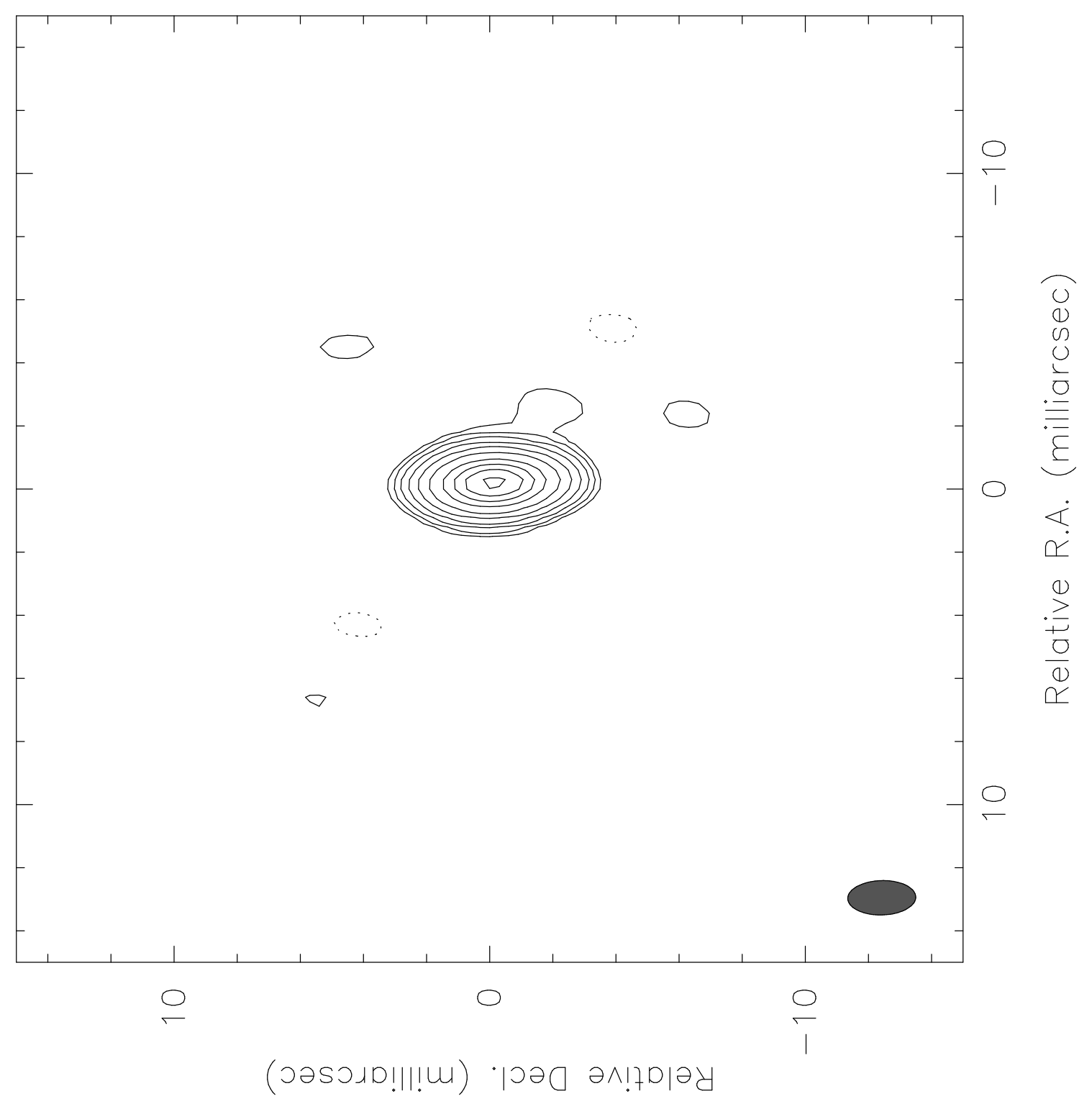




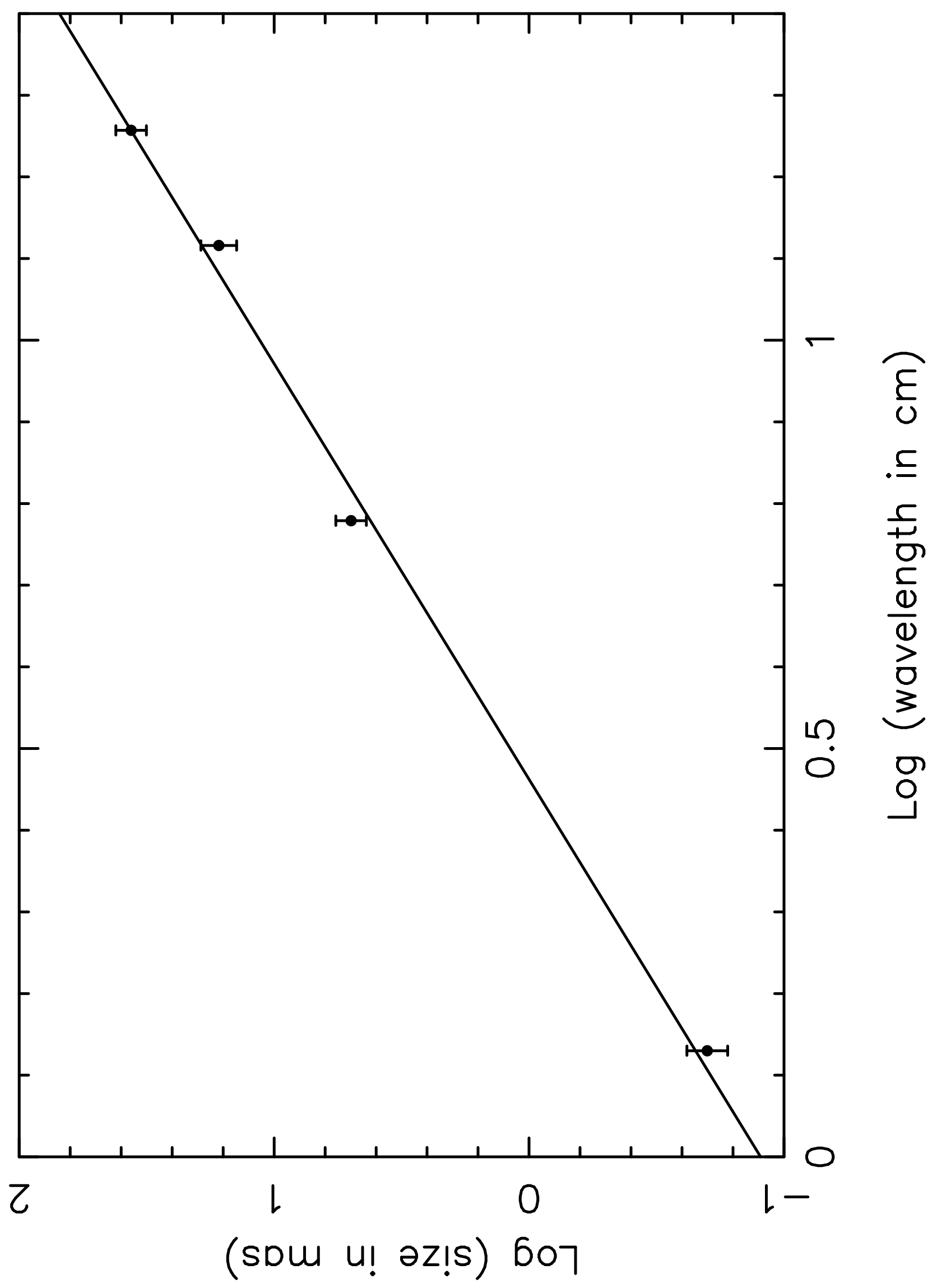

